\title{
Robust Adaptive Beamforming for Multiple Signals of Interest with Cycle Frequency Error
}

\author{
Ju-Hong Lee ${ }^{1}$ and Chia-Cheng Huang ${ }^{2}$ \\ ${ }^{1}$ Department of Electrical Engineering, Graduate Institute of Communication Engineering, and \\ Graduate Institute of Biomedical Electronics and Bioinformatics, National Taiwan University, Taipei 10617, Taiwan \\ ${ }^{2}$ Graduate Institute of Communication Engineering, National Taiwan University, Taipei 10617, Taiwan
}

Correspondence should be addressed to Ju-Hong Lee, juhong@cc.ee.ntu.edu.tw

Received 3 September 2010; Revised 6 December 2010; Accepted 10 December 2010

Academic Editor: Enrico Capobianco

Copyright (C) 2010 J.-H. Lee and C.-C. Huang. This is an open access article distributed under the Creative Commons Attribution License, which permits unrestricted use, distribution, and reproduction in any medium, provided the original work is properly cited.

This paper deals with the problem of robust adaptive array beamforming by exploiting the signal cyclostationarity. Recently, a novel cyclostationarity-exploiting beamforming method has been proposed by J.-H. Lee and C.-C. Huang (2009) for dealing with the situation of multiple signals of interest (SOI) based on the LS-SCORE algorithm. This method is referred to as the multiple LSSCORE (MLS-SCORE) algorithm. However, the MLS-SCORE algorithm suffers from severe performance degradation even if there is a small mismatch in the cycle frequencies of the SOIs. In this paper, we evaluate the performance of the MLS-SCORE algorithm in the presence of cycle frequency error (CFE). The output SINR of an adaptive beamforming using the MLS-SCORE algorithm deteriorates like a sinc function as the number of data snapshots increases. To tackle this difficulty, we present an efficient method to find an appropriate estimate for each of the cycle frequencies of the SOIs iteratively to achieve robust adaptive beamforming against the CFE. Simulation results for showing the effectiveness of the proposed method are provided.

\section{Introduction}

For conventional array beamforming, the a priori information required for adapting the weights is either the direction vector or the waveform of the signal of interest (SOI) [1]. A signal with cyclostationarity has the statistical property of correlating with either a frequency-shift (referred to as spectral self-coherence) or a complex-conjugate version (referred to as spectral conjugate self-coherence) of itself. For example, spectral self-coherence is induced at multiples of the symbol rate in PCM signals and spectral conjugate self-coherence is commonly induced at twice the carrier frequency in BPSK signals $[2,3]$. By restoring those properties at a known value of frequency separation, it is possible to extract the SOI and suppress the signals not of interest (SNOI) and noise. Adaptive beamforming utilizing signal cyclostationarity has been widely considered [3-5]. These cyclostationarity-exploiting techniques do not need training signals, the knowledge of array manifold, or noise characteristics. The least-square spectral self-coherent restoral (LS-SCORE) algorithm has been presented by [3] to deal with the problem of blind adaptive signal extraction. As the number of data snapshots approaches infinity, it has been shown in [3] that the performance of the LS-SCORE algorithm approaches that of the conventional beamforming methods developed by maximizing the output signal-to-interference plus noise ratio (SINR). The a priori information required by the LS-SCORE algorithm is only the cycle frequency of the SOI. Hence, its performance is sensitive to the accuracy of the presumed cycle frequency. However, the actual cycle frequency may not be known very well in some applications due to, for example, the phenomenon of Doppler shift. Accordingly, several existing works deal with the cycle frequency error (CFE) have been presented by [6-8]. The robust cyclostationarityexploiting beamforming methods presented by $[6,7]$ are in conjunction with the SCORE algorithms. These methods developed an iterative procedure to find an appropriate estimate of the cycle frequency of the SOI. Recently, a robust cyclostationarity-exploiting direction-finding approach [8] adopts the average of the cyclic correlation matrices in a presumed range of cycle frequencies including the actual one to alleviate the performance degradation due to the CFE. 
Nevertheless, the aforementioned methods consider the case of one SOI with CFE.

For many practical applications, such as satellite communications [9], an antenna array is required to possess the beamforming capability that receives more than one SOI with specified gain constraints while suppressing all SNOI. This goal can be achieved using an antenna array with a multiple-beam pattern $[9,10]$. Recently, a novel cyclostationarity-exploiting beamforming method has been proposed by [11] for dealing with the situation of multiple SOIs based on the LS-SCORE algorithm. This approach is referred to as the multiple LS-SCORE (MLS-SCORE) algorithm. It has been shown in [11] that the solution of the MLS-SCORE algorithm converges to the solution of the conventional linearly constrained minimum variance (LCMV) as the number of data snapshots approaches infinity. In this paper, we first evaluate the performance of the MLS-SCORE algorithm in the presence of CFE. This results in an analytical formula that demonstrates the behavior of the performance degradation for the MLSSCORE algorithm. It is shown that the output SINR of an adaptive array beamformer using the MLS-SCORE algorithm deteriorates like a sinc function as the number of data snapshots increases. To overcome the CFE difficulty, we then develop an efficient method to formulate the problem as an optimization problem for reducing the CFE of the SOIs iteratively. Finally, the convergence property of the proposed method is evaluated. The effectiveness of the proposed method is demonstrated by several simulation examples. It is shown that the proposed method can effectively cope with the performance degradation for the MLS-SCORE algorithm to achieve robust capability against the CFE.

This paper is organized as follows: in Section 2, we briefly describe the property of signal cyclostationarity, the MLS-SCORE algorithm [11], and the performance analysis of the MLS-SCORE algorithms under CFE. The proposed method is presented in Section 3. The convergence analysis of the proposed method is presented in Section 4. Simulation examples for confirming the effectiveness of the proposed method are provided in Section 5. Finally, we conclude the paper in Section 6.

\section{Preliminaries}

2.1. Signal Cyclostationarity. Many man-made communication signals exhibit cyclostationarity with cycle frequency equal to the twice of the carrier frequency or multiples of the baud rate or combinations of these [2]. According to [2], a signal $s(t)$ is said to possess the second-order periodicity with cycle frequency $\alpha$ if and only if the cyclic or the cyclic conjugate autocorrelation function given by

$$
r_{s s}(\alpha, \tau) \equiv \lim _{T \rightarrow \infty}\left\langle s(t) s^{*}(t-\tau) e^{-j 2 \pi \alpha t}\right\rangle_{T},
$$

or

$$
r_{s s^{*}}(\alpha, \tau) \equiv \lim _{T \rightarrow \infty}\left\langle s(t) s(t-\tau) e^{-j 2 \pi \alpha t}\right\rangle_{T}
$$

does not equal zero at cycle frequency $\alpha$ for some time delay $\tau$, where the superscript “ $*$ " denotes the complex conjugate and $\langle\cdot\rangle_{T} \equiv(1 / T) \int_{T} \cdot d t$ represents the finite-time average operation. Moreover, $s(t)$ is addressed as possessing the selfcoherent or conjugate self-coherent properties for $r_{s s}(\alpha, \tau)$ or $r_{s s^{*}}(\alpha, \tau)$ does not equal zero at cycle frequency $\alpha$. In matrix form, the cyclic conjugate autocorrelation matrix associated with the data vector $\mathbf{x}(t)$ can be expressed as

$$
\mathbf{R}_{x x^{*}}(\alpha, \tau) \equiv \lim _{T \rightarrow \infty}\left\langle\mathbf{x}(t) \mathbf{x}^{T}(t-\tau) e^{-j 2 \pi \alpha t}\right\rangle_{T},
$$

where the superscript " $T$ " denotes the transpose operation.

2.2. The MLS-SCORE Algorithm. Consider that there are total $(K+d)$ far-field narrowband signals including $d$ SOIs and $K$ SNOI impinging on an $M$-element antenna array. Assume that the background noise is spatially white. The received data vector $\mathbf{x}(t)$ is given by [1]

$$
\mathbf{x}(t)=\sum_{i=1}^{d} \mathbf{a}_{i} s_{i}(t)+\sum_{k=1}^{K} \mathbf{b}_{k} j_{k}(t)+\mathbf{n}(t)=\sum_{i=1}^{d} \mathbf{a}_{i} s_{i}(t)+\mathbf{v}(t),
$$

where $s_{i}(t)$ and $j_{k}(t)$ are the waveforms of the $i$ th SOI and the $k$ th SNOI, $a_{i}$ and $b_{k}$ represent the direction vectors of the $i$ th SOI and the $k$ th SNOI, and $\mathbf{v}(t)$ includes the SNOI and noise, respectively.

Without loss of generality, we suppose that the SOIs are cyclostationary and have the cycle frequencies $\underline{\alpha}$, where $\underline{\alpha}=\left\{\alpha_{i} \mid i=1,2, \ldots, d\right\}$ denotes the set of cycle frequencies of the SOIs. However, $\mathbf{v}(t)$ is not cyclostationary at $\underline{\alpha}$ and is temporally uncorrelated with the SOIs. Based on the MLSSCORE algorithm [11], the optimal weight vector is given by

$$
\hat{\mathbf{w}}_{\mathrm{mls}}(\underline{\alpha})=\arg \min _{\mathbf{w}}\left\langle|y(t)-z(\underline{\alpha}, t)|^{2}\right\rangle_{T},
$$

where the array output $y(t)=\mathbf{w}^{H} \mathbf{x}(t)$, and the reference signal $z(\underline{\alpha}, t)$ is given by

$$
z(\underline{\alpha}, t)=\mathbf{c}^{H} \mathbf{u}(\underline{\alpha}, t)=\mathbf{c}^{H} \mathbf{x}^{*}(t-\tau) \sum_{i=1}^{d} e^{j 2 \pi \alpha_{i} t},
$$

where $\mathbf{u}(\underline{\alpha}, t)=\mathbf{x}^{*}(t-\tau) \sum_{i=1}^{d} e^{j 2 \pi \alpha_{i} t}$ denotes the control signal and $\mathbf{c}$ a fixed control vector. Moreover, the superscript " $H$ " denotes the conjugate transpose. Let the sampling interval be $T_{s}$. The optimal weight vector of (5) with $n$ data snapshots (i.e., $T=n T_{s}$ ) used is given by

$$
\widehat{\mathbf{w}}_{\mathrm{mls}}(\underline{\alpha}, n)=\hat{\mathbf{R}}_{x x}^{-1}(n) \hat{\mathbf{r}}_{x z}(\underline{\alpha}, n),
$$

where $\hat{\mathbf{R}}_{x x}(n)=\left\langle\mathbf{x}(t) \mathbf{x}^{H}(t)\right\rangle_{T}$ and $\widehat{\mathbf{r}}_{x z}(\underline{\alpha}, n)=\left\langle\mathbf{x}(t) z^{*}(\underline{\alpha}, t)\right\rangle_{T}$ are the sample autocorrelation matrix of $\mathbf{x}(t)$ and the sample cross-correlation vector of $\mathbf{x}(t)$ and $z(\underline{\alpha}, t)$, respectively. It has been shown in [11] that $\hat{\mathbf{w}}_{\mathrm{mls}}(\underline{\alpha}, n)$ converges to the solution of the conventional optimum array beamforming based on the linearly constrained minimum variance (LCMV) criterion when $T$ approaches infinity. We note from (4) and (6) that $\widehat{\mathbf{r}}_{x z}(\underline{\alpha}, n)=\hat{\mathbf{R}}_{x u}(\underline{\alpha}, n)$ c, where the sample cross-correlation matrix $\widehat{\mathbf{R}}_{x u}(\underline{\alpha}, n)$ is defined as

$$
\hat{\mathbf{R}}_{x u}(\underline{\alpha}, n)=\left\langle\mathbf{x}(t) \mathbf{u}^{H}(\underline{\alpha}, t)\right\rangle_{T}=\sum_{i=1}^{d} \widehat{\mathbf{R}}_{x x^{*}}\left(\alpha_{i}, \tau\right),
$$


where $\hat{\mathbf{R}}_{x x^{*}}\left(\alpha_{i}, \tau\right)=\left\langle\mathbf{x}(t) \mathbf{x}^{T}(t-\tau) e^{-j 2 \pi \alpha_{i} t}\right\rangle_{T}$ is the sample cyclic conjugate autocorrelation matrix of $\mathbf{x}(t)$. We observe from (7) that the a priori information required for computing the optimal vector $\widehat{\mathbf{w}}_{\mathrm{mls}}(\underline{\alpha}, n)$ is only the cycle frequencies of the SOIs. Hence, the performance of the MLS-SCORE algorithm is sensitive to the accuracy of the presumed cycle frequency for each of the SOIs. However, the actual cycle frequency may not be known very well in some applications due to, for example, the phenomenon of Doppler shift. Next, we evaluate the performance of the MLS-SCORE algorithm in the presence of CFE.

2.3. Performance Analysis under CFE. From (8), the sample cyclic conjugate correlation matrix at the presumed frequencies $\underline{\hat{\alpha}}$ is approximately equal to

$$
\hat{\mathbf{R}}_{x u}(\underline{\hat{\alpha}}, n) \approx \sum_{i=1}^{d} \hat{r}_{s_{i} *_{i}^{*}}\left(\hat{\alpha}_{i}, \tau\right) \mathbf{a}_{i} \mathbf{a}_{i}^{T}+\hat{\mathbf{R}}_{v v^{*}}(\underline{\hat{\alpha}}, \tau)
$$

as the number of data snapshots is large enough, where $\hat{r}_{s_{i} s_{i}^{*}}\left(\hat{\alpha}_{i}, \tau\right)=\left\langle s_{i}(t) s_{i}(t-\tau) e^{-j 2 \pi \hat{\alpha}_{i} t}\right\rangle_{T}$ is the sample cyclic conjugate autocorrelation function of the $i$ th SOI, and $\hat{\mathbf{R}}_{v v^{*}}(\underline{\hat{\alpha}}, \tau)=\left\langle\mathbf{v}(t) \mathbf{v}^{T}(t-\tau) \sum_{i=1}^{d} e^{-j 2 \pi \hat{\alpha}_{i} t}\right\rangle_{T}$ denotes the sample cyclic conjugate autocorrelation matrix of $\mathbf{v}(t)$. In fact, $\hat{\mathbf{R}}_{x u}(\underline{\hat{\alpha}}, n)$ also includes the sample cyclic cross-correlations between the SOIs, the SOIs and the SNOI, the SOIs and noise, and the SNOI and noise. However, they are negligible when $T$ is large enough. Due to the fact that the cyclic spectrum of a cyclostationary signal is discrete in the cycle frequency, the cyclic conjugate correlation function of the $i$ th SOI is given by

$$
r_{s_{i} s_{i}^{*}}\left(\hat{\alpha}_{i}, \tau\right)=\sum_{l} \rho_{i, l}(\tau) \delta\left(\hat{\alpha}_{i}-\alpha_{i, l}\right),
$$

where $\alpha_{i, l}$ 's are the cycle frequencies of $i$ th SOI, $\rho_{i, l}(\tau)$ denotes the strength of the $i$ th SOI at cycle frequency $\alpha_{i, l}$, and $\delta(\cdot)$ represents the Kronecker delta. According to (10), the sample cyclic conjugate autocorrelation function of the $i$ th SOI is given by

$$
\hat{r}_{s_{i} s_{i}^{*}}\left(\hat{\alpha}_{i}, \tau\right) \approx \sum_{l} \hat{\rho}_{i, l}(\tau) \operatorname{sinc}\left(\left(\hat{\alpha}_{i}-\alpha_{i, l}\right) T\right),
$$

where $\operatorname{sinc}(x)=(\sin (\pi x)) / \pi x$ is a sinc function. Substituting (11) into (9) yields

$$
\hat{\mathbf{R}}_{x u}(\underline{\hat{\alpha}}, n) \approx \sum_{i=1}^{d} \sum_{l} \hat{\rho}_{i, l}(\tau) \operatorname{sinc}\left(\left(\hat{\alpha}_{i}-\alpha_{i, l}\right) T\right) \mathbf{a}_{i} \mathbf{a}_{i}^{T}+\hat{\mathbf{R}}_{v v^{*}}(\underline{\hat{\alpha}}, \tau) .
$$

Let the presumed cycle frequency of the $i$ th SOI be denoted by $\hat{\alpha}_{i}=\alpha_{i, 1}+\Delta \alpha_{i}$ (i.e., $\underline{\hat{\alpha}}=\underline{\alpha}+\Delta \underline{\alpha}$ ), where $\alpha_{i, 1}$ and $\Delta \alpha_{i}$ represent the actual cycle frequency and the amount of CFE of the $i$ th SOI, respectively. Then, (12) becomes

$$
\begin{aligned}
\hat{\mathbf{R}}_{x u}(\underline{\hat{\alpha}}, n) \approx & \sum_{i=1}^{d}\left\{\hat{\rho}_{i, 1}(\tau) \operatorname{sinc}\left(\Delta \alpha_{i} T\right)+\sum_{l \neq 1} \hat{\rho}_{i, l}(\tau) \operatorname{sinc}\left(\tilde{\alpha}_{i, l} T\right)\right\} \\
& \times \mathbf{a}_{i} \mathbf{a}_{i}^{T}+\hat{\mathbf{R}}_{v v^{*}}(\underline{\hat{\alpha}}, \tau)
\end{aligned}
$$

with $\tilde{\alpha}_{i, l}=\alpha_{i, 1}-\alpha_{i, l}+\Delta \alpha_{i}$. Equation (13) reveals that

$$
\begin{aligned}
\hat{\mathbf{r}}_{x z}(\underline{\hat{\alpha}}, n) \approx & \sum_{i=1}^{d}\left\{\hat{\rho}_{i, 1}(\tau) \operatorname{sinc}\left(\Delta \alpha_{i} T\right)+\sum_{l \neq 1} \hat{\rho}_{i, l}(\tau) \operatorname{sinc}\left(\tilde{\alpha}_{i, l} T\right)\right\} \\
& \times\left(\mathbf{c}^{H} \mathbf{a}_{i}^{*}\right) \mathbf{a}_{i}+\hat{\mathbf{R}}_{v v^{*}}(\underline{\hat{\alpha}}, \tau) \mathbf{c} .
\end{aligned}
$$

From (14), we note that the effect of the SNOI and noise is negligible when $\Delta \alpha_{i}=0$. Accordingly, $\hat{\mathbf{r}}_{x z}(\underline{\hat{\alpha}}, n)$ is approximately equal to $\sum_{i=1}^{d} \gamma_{i} \mathbf{a}_{i}$, where $\gamma_{i}=\hat{\rho}_{i, 1}\left(\mathbf{c}^{H} \mathbf{a}_{i}^{*}\right)$ is a constant. It has been shown by [11] that $\hat{\mathbf{w}}_{\mathrm{mls}}(\underline{\alpha}, n)$ of $(7)$ converges to the LCMV beamformer as long as $\mathbf{c}^{\bar{H}} \mathbf{a}_{i}^{*} \neq 0$ for all $i$. On the contrary, when $\Delta \alpha_{i} \neq 0$, we note that there exists the effects of cycle leakage on $\hat{\mathbf{r}}_{x z}(\underline{\hat{\alpha}}, n)$ through a sinc window due to the fact that $\operatorname{sinc}\left(\Delta \alpha_{i} T\right)=0$ when $\Delta \alpha_{\mathrm{i}} T$ equals to an integer or $T$ approaches infinity. Consequently, the output SINR of an adaptive array beamformer based on the MLSSCORE algorithm exists periodic nulls as the number of data snapshots increases. This leads to performance degradation for the MLS-SCORE algorithm.

\section{The Proposed Method}

From (14), we observe that both of the $\operatorname{sinc}\left(\Delta \alpha_{i} T\right)$ and $\operatorname{sinc}\left(\tilde{\alpha}_{i, l} T\right)$ approach zero as $T$ increases when CFE exists. Hence, the performance degradation for the MLS-SCORE algorithm becomes severer as $T$ increases. To overcome the difficulty, we present an efficient method in conjunction with the MLS-SCORE algorithm to find an appropriate estimate for $\alpha_{i}, i=1,2, \ldots, d$.

First, we derive a squared error based on the solution of the MLS-SCORE algorithm shown by (7) and replace $\hat{\mathbf{w}}_{\mathrm{mls}}(\underline{\alpha}, n)$ with $\hat{\mathbf{w}}_{\mathrm{mls}}(\underline{\hat{\alpha}}, n)$ to obtain

$$
\begin{aligned}
J_{\mathrm{mls}}= & \left\langle\left|y_{1}(t)-z(\underline{\hat{\alpha}}, t)\right|^{2}\right\rangle_{T} \\
= & \hat{\mathbf{w}}_{\mathrm{mls}}^{H}(\underline{\hat{\alpha}}, n) \hat{\mathbf{R}}_{x x}(n) \hat{\mathbf{w}}_{\mathrm{mls}}(\underline{\hat{\alpha}}, n)-\mathbf{c}^{H} \hat{\mathbf{R}}_{x u}^{H}(\underline{\hat{\alpha}}, n) \hat{\mathbf{w}}_{\mathrm{mls}}(\underline{\hat{\alpha}}, n) \\
& -\hat{\mathbf{w}}_{\mathrm{mls}}^{H}(\underline{\hat{\alpha}}, n) \hat{\mathbf{R}}_{x u}(\underline{\hat{\alpha}}, n) \mathbf{c}+\mathbf{c}^{H} \hat{\mathbf{R}}_{x x}^{*}(n) \mathbf{c} \\
= & \mathbf{c}^{H} \hat{\mathbf{R}}_{x x}^{*}(n) \mathbf{c}-\hat{\mathbf{w}}_{\mathrm{mls}}^{H}(\underline{\hat{\alpha}}, n) \hat{\mathbf{R}}_{x u}(\underline{\hat{\alpha}}, n) \mathbf{c},
\end{aligned}
$$

where $y_{1}(t)=\widehat{\mathbf{w}}_{\mathrm{mls}}^{H}(\underline{\hat{\alpha}}, n) \mathbf{x}(t)$. We note that $J_{\mathrm{mls}}$ of (15) is greater than or equal to zero. Hence, minimizing $J_{\mathrm{mls}}$ is equivalent to maximizing the second term of (15) which is redefined as follows:

$$
C(\underline{\hat{\alpha}}, n)=\hat{\mathbf{w}}_{\mathrm{mls}}^{H}(\underline{\hat{\alpha}}, n) \hat{\mathbf{R}}_{x u}(\underline{\hat{\alpha}}, n) \mathbf{c} .
$$

We note that the objective function $C(\underline{\hat{\alpha}}, n)$ achieves its maximum when $\underline{\alpha}=\underline{\hat{\alpha}}$ and decays to zero as the number of snapshots increases when $\underline{\alpha} \neq \underline{\hat{\alpha}}$. Therefore, (16) can be used as an objective function for formulating an optimization problem. Then, we resort to solving the following optimization problem:

$$
\max _{\underline{\underline{\alpha}}} C(\underline{\hat{\alpha}}, n) .
$$


Using the steepest descent method for solving (17), we take the derivative of $C(\underline{\hat{\alpha}}, n)$ with respect to $\underline{\hat{\alpha}}$ as follows:

$$
\begin{aligned}
\nabla_{\underline{\hat{\alpha}}} C(\underline{\hat{\alpha}}, n)= & \frac{\partial}{\partial \underline{\hat{\alpha}}}\left\{\hat{\mathbf{w}}_{\mathrm{mls}}^{H}(\underline{\hat{\alpha}}, n) \hat{\mathbf{R}}_{x u}(\underline{\hat{\alpha}}, n) \mathbf{c}\right\} \\
= & \mathbf{c}^{H} \frac{\partial \hat{\mathbf{R}}_{x u}^{H}(\underline{\hat{\alpha}}, n)}{\partial \underline{\hat{\alpha}}} \hat{\mathbf{R}}_{x x}^{-1}(n) \hat{\mathbf{R}}_{x u}(\underline{\hat{\alpha}}, n) \mathbf{c} \\
& +\mathbf{c}^{H} \hat{\mathbf{R}}_{x u}^{H}(\underline{\hat{\alpha}}, n) \hat{\mathbf{R}}_{x x}^{-1}(n) \frac{\partial \hat{\mathbf{R}}_{x u}(\underline{\hat{\alpha}}, n)}{\partial \underline{\hat{\alpha}}} \mathbf{c} \\
= & 2 \Re\left\{\mathbf{c}^{H} \frac{\partial \hat{\mathbf{R}}_{x u}^{H}(\underline{\hat{\alpha}}, n)}{\partial \underline{\hat{\alpha}}} \hat{\mathbf{R}}_{x x}^{-1}(n) \hat{\mathbf{R}}_{x u}(\underline{\hat{\alpha}}, n) \mathbf{c}\right\} \\
= & 2 \Re\left\{\mathbf{c}^{H} \hat{\mathbf{R}}_{x u}^{\prime H}(\underline{\hat{\alpha}}, n) \hat{\mathbf{w}}_{\mathrm{mls}}(\underline{\hat{\alpha}}, n)\right\},
\end{aligned}
$$

where $\mathfrak{R}\{y\}$ denotes the real part of $y$ and $\hat{\mathbf{R}}_{x u}^{\prime}(\underline{\hat{\alpha}}, n)=$ $\left(\partial \hat{\mathbf{R}}_{x u}(\underline{\hat{\alpha}}, n)\right) / \partial \underline{\hat{\alpha}}$. In vector form, we can rewrite $C(\underline{\hat{\alpha}}, n)$ of (18) as follows:

$$
\left[\begin{array}{c}
\nabla_{\hat{\alpha}_{1}} C(\underline{\hat{\alpha}}, n) \\
\nabla_{\hat{\alpha}_{2}} C(\underline{\hat{\alpha}}, n) \\
\vdots \\
\nabla_{\hat{\alpha}_{d}} C(\underline{\hat{\alpha}}, n)
\end{array}\right]=\left[\begin{array}{c}
2 \Re\left\{\mathbf{c}^{H} \hat{\mathbf{R}}_{x u}^{\prime H}\left(\hat{\alpha}_{1}, n\right) \widehat{\mathbf{w}}_{\mathrm{mls}}(\underline{\hat{\alpha}}, n)\right\} \\
2 \mathfrak{R}\left\{\mathbf{c}^{H} \hat{\mathbf{R}}_{x u}^{\prime H}\left(\hat{\alpha}_{2}, n\right) \hat{\mathbf{w}}_{\mathrm{mls}}(\underline{\hat{\alpha}}, n)\right\} \\
\vdots \\
2 \mathfrak{R}\left\{\mathbf{c}^{H} \hat{\mathbf{R}}_{x u}^{\prime H}\left(\hat{\alpha}_{d}, n\right) \hat{\mathbf{w}}_{\mathrm{mls}}(\underline{\hat{\alpha}}, n)\right\}
\end{array}\right]
$$

with $\hat{\mathbf{R}}_{x u}^{\prime}\left(\hat{\alpha}_{i}, n\right)=\left(\partial \hat{\mathbf{R}}_{x u}(\underline{\hat{\alpha}}, n)\right) / \partial \hat{\alpha}_{i}, i=1,2, \ldots, d$, is given by

$$
\hat{\mathbf{R}}_{x u}^{\prime}\left(\hat{\alpha}_{i}, n\right)=\left\langle\mathbf{x}(t) \mathbf{x}^{T}(t-\tau) e^{-j 2 \pi \hat{\alpha}_{i} t}(-j 2 \pi t)\right\rangle_{T} .
$$

Accordingly, we update $\underline{\hat{\alpha}}$ at the time instant $(n+1)$ as follows:

$$
\underline{\hat{\alpha}}(n+1)=\underline{\hat{\alpha}}(n)+\left.\boldsymbol{\Phi}(n) \nabla_{\underline{\hat{\alpha}}} C(\underline{\hat{\alpha}}, n)\right|_{\hat{\alpha}=\hat{\alpha}(n)},
$$

where $\Phi(n)=\operatorname{diag}\left\{\mu_{1}(n), \mu_{2}(n), \ldots, \mu_{d}(n)\right\}$ is a diagonal matrix. The diagonal entries $\mu_{i}(n), i=1,2, \ldots, d$, are positive real-valued parameters referred to as the step-size parameters. To ensure the convergence of the steepestdescent algorithm used by (21), we set the ith step-size parameter equal to

$$
\mu_{i}(n)=\left\{\lambda_{\max }\left\{\hat{\mathbf{R}}_{x u}^{\prime}\left(\hat{\alpha}_{i}(n), n\right)\right\}\right\}^{-P_{i}}
$$

where $\lambda_{\max }\{\mathbf{R}\}$ denotes the maximum singular value of the matrix $\mathbf{R}$, and $P_{i}$ is the appropriate positive real value determined by experiment. Substituting $\underline{\hat{\alpha}}(n+1)$ of $(21)$ into (7), the corresponding optimal weight vector at the time instant $(n+1)$ is given by

$$
\widehat{\mathbf{w}}_{\mathrm{mls}}(\underline{\hat{\alpha}}(n+1), n+1)=\widehat{\mathbf{R}}_{x x}^{-1}(n+1) \widehat{\mathbf{r}}_{x z}(\underline{\hat{\alpha}}(n+1), n+1) .
$$

For practical implementation, we compute the required sample correlation matrix $\widehat{\mathbf{R}}_{x x}(n)$ and the cross-correlation vector $\hat{\mathbf{r}}_{x z}(\underline{\hat{\alpha}}(n), n)$ by utilizing $(24)$, where $\mathbf{x}(n)=\mathbf{x}\left(n T_{s}\right)$ and $L_{0}$ is a preset positive integer. Since $\underline{\hat{\alpha}}(n)$ becomes more appropriate as $n$ increases, we use $L_{0}$ data snapshots to update the corresponding correlations for increasing the effect on the estimates of the considered correlations shown by (24)

$$
\begin{gathered}
\hat{\mathbf{R}}_{x x}(n)=\left(1-\frac{1}{n}\right) \hat{\mathbf{R}}_{x x}(n-1)+\frac{1}{n} \mathbf{x}(n) \mathbf{x}^{H}(n) \\
\hat{\mathbf{r}}_{x z}(\underline{\hat{\alpha}}(n), n)= \begin{cases}\frac{1}{n} \sum_{l=1}^{n} \mathbf{x}(l) z^{*}(\underline{\hat{\alpha}}(l), l), & \text { for } n \leq L_{0} \\
\left(1-\frac{1}{n}\right) \hat{\mathbf{r}}_{x z}(\underline{\hat{\alpha}}(n-1), n-1) & \\
+\frac{1}{n} \sum_{l=n-L_{0}+1}^{n} \mathbf{x}(l) z^{*}(\underline{\hat{\alpha}}(l), l), & \text { for } n>L_{0}\end{cases}
\end{gathered}
$$

\section{Convergence Analysis}

Here, the convergence property of the proposed method is evaluated. For simplicity, we set $\tau=0$. The objective function $C(\underline{\hat{\alpha}}, n)$ of $(16)$ can be rewritten as

$$
\begin{aligned}
C(\underline{\hat{\alpha}}, n)= & \mathbf{c}^{H} \hat{\mathbf{R}}_{x u}^{H}(\underline{\hat{\alpha}}, n) \hat{\mathbf{R}}_{x x}^{-1}(n) \hat{\mathbf{R}}_{x u}(\underline{\hat{\alpha}}, n) \mathbf{c} \\
= & \mathbf{c}^{H}\left\{\frac{1}{n} \sum_{l=1}^{n} \mathbf{x}^{*}(l) \mathbf{x}^{H}(l) \sum_{i=1}^{d} e^{j 2 \pi \hat{\alpha}_{i} l T_{s}}\right\} \hat{\mathbf{R}}_{x x}^{-1}(n) \\
& \times\left\{\frac{1}{n} \sum_{k=1}^{n} \mathbf{x}(k) \mathbf{x}^{T}(k) \sum_{m=1}^{d} e^{-j 2 \pi \hat{\alpha}_{m} k T_{s}}\right\} \mathbf{c} \\
\approx & \frac{1}{n^{2}} \sum_{i=1}^{d} \sum_{m=1}^{d} \sum_{l=1}^{n} \sum_{k=1}^{n}\{\underbrace{\mathbf{c}^{H} \mathbf{x}^{*}(l) \mathbf{x}^{T}(k) \mathbf{c}}_{A_{1}}\} \\
& \times\{\underbrace{\mathbf{x}^{H}(l) \hat{\mathbf{R}}_{x x}^{-1}(n) \mathbf{x}(k)}_{A_{2}}\} e^{-j 2 \pi\left(k \hat{\alpha}_{m}-l \hat{\alpha}_{i}\right) T_{s},},
\end{aligned}
$$

where the term $A_{1}$ in (25) can be expressed as

$$
\begin{aligned}
A_{1} & =\mathbf{c}^{H}\left\{\sum_{i=1}^{d} \mathbf{a}_{i} s_{i}(l)+\mathbf{v}(l)\right\}^{*}\left\{\sum_{m=1}^{d} \mathbf{a}_{m} s_{m}(k)+\mathbf{v}(k)\right\}^{T} \mathbf{c} \\
& \approx \sum_{i=1}^{d} s_{i}^{*}(l) s_{i}(k)\left\{\mathbf{c}^{H} \mathbf{a}_{i}^{*} \mathbf{a}_{i}^{T} \mathbf{c}\right\}+\mathbf{c}^{H} \mathbf{v}^{*}(l) \mathbf{v}^{T}(k) \mathbf{c} .
\end{aligned}
$$

We note from (26) that the cross-terms disappear due to the assumed uncorrelation between $s_{i}(t)$ and $s_{m}(t)$. As to the term $A_{2}$, we have

$$
\begin{aligned}
A_{2} & \approx \sum_{i=1}^{d} \sum_{m=1}^{d} s_{i}^{*}(l) s_{m}(k)\left\{\mathbf{a}_{i}^{H} \hat{\mathbf{R}}_{x x}^{-1}(n) \mathbf{a}_{m}\right\}+\mathbf{v}^{H}(l) \hat{\mathbf{R}}_{x x}^{-1}(n) \mathbf{v}(k) \\
& \approx \sum_{i=1}^{d} s_{i}^{*}(l) s_{i}(k)\left\{\mathbf{a}_{i}^{H} \hat{\mathbf{R}}_{x x}^{-1}(n) \mathbf{a}_{i}\right\}+\mathbf{v}^{H}(l) \hat{\mathbf{R}}_{x x}^{-1}(n) \mathbf{v}(k) .
\end{aligned}
$$


The approximation is obtained due to $\mathbf{a}_{i}^{H} \hat{\mathbf{R}}_{x x}^{-1}(n) a_{m} \approx 0$ when $i \neq m$ as the number of data snapshots $n$ is large enough. According to (26) and (27), we have

$$
\begin{aligned}
A_{1} A_{2} \approx & \sum_{i=1}^{d}\left\{s_{i}^{2}(l)\right\}^{*} s_{i}^{2}(k)\left\{\mathbf{a}_{i}^{H} \hat{\mathbf{R}}_{x x}^{-1}(n) \mathbf{a}_{i}\right\}\left|\mathbf{c}^{H} \mathbf{a}_{i}^{*}\right|^{2} \\
& +\mathbf{c}^{H} \mathbf{v}^{*}(l) \mathbf{v}^{H}(l) \hat{\mathbf{R}}_{x x}^{-1}(n) \mathbf{v}(k) \mathbf{v}^{T}(k) \mathbf{c} .
\end{aligned}
$$

Substituting (28) into (25), we obtain an approximation for $C(\underline{\hat{\alpha}}, n)$ as follows:

$$
\begin{aligned}
C(\underline{\hat{\alpha}}, n) \approx & \frac{1}{n^{2}} \sum_{i=1}^{d} \sum_{l=1}^{n} \sum_{k=1}^{n}\left\{\left\{s_{i}^{2}(l) e^{-j 2 \pi \hat{\alpha}_{i} l T_{s}}\right\}^{*}\left\{s_{i}^{2}(k) e^{-j 2 \pi \hat{\alpha}_{i} k T_{s}}\right\}\right. \\
& \times\left\{\mathbf{a}_{i}^{H} \hat{\mathbf{R}}_{x x}^{-1}(n) \mathbf{a}_{i}\right\}\left|\mathbf{c}^{H} \mathbf{a}_{i}^{*}\right|^{2} \\
& +\mathbf{c}^{H}\left\{\mathbf{v}(l) \mathbf{v}^{T}(l) \sum_{i=1}^{d} e^{j 2 \pi \hat{\alpha}_{i} l T_{s}}\right\}^{*} \\
& \left.\times \hat{\mathbf{R}}_{x x}^{-1}(n)\left\{\mathbf{v}(k) \mathbf{v}^{T}(k) \sum_{i=1}^{d} e^{j 2 \pi \hat{\alpha}_{i} k T_{s}}\right\} \mathbf{c}\right\} \\
\approx & \sum_{i=1}^{d}\left|\hat{r}_{s_{i} s_{i}^{*}}\left(\hat{\alpha}_{i}, 0\right)\right|^{2}\left\{\mathbf{a}_{i}^{H} \hat{\mathbf{R}}_{x x}^{-1}(n) \mathbf{a}_{i}\right\}\left|\mathbf{c}^{H} \mathbf{a}_{i}^{*}\right|^{2} \mathbf{c}^{H} \\
& \times \hat{\mathbf{R}}_{v v^{*}}^{H}(\underline{\hat{\alpha}}, 0) \hat{\mathbf{R}}_{x x}^{-1}(n) \hat{\mathbf{R}}_{v v^{*}}(\underline{\hat{\alpha}}, 0) \mathbf{c} .
\end{aligned}
$$

We note from (29) that the last term vanishes asymptotically due to $\underline{\hat{\alpha}}$ (note that $\underline{\hat{\alpha}}=\underline{\hat{\alpha}}(n)$ ) becomes more appropriate as $n$ increases. Accordingly, the $i$ th entry $\nabla_{\hat{\alpha}_{i}} C(\underline{\hat{\alpha}}, n)$ in (19) can be further expressed as

$$
\nabla_{\widehat{\alpha}_{i}} C(\underline{\hat{\alpha}}, n) \approx \frac{\partial\left|\hat{r}_{s_{i} *_{i}^{*}}\left(\hat{\alpha}_{i}, 0\right)\right|^{2}}{\partial \hat{\alpha}_{i}}\left\{\mathbf{a}_{i}^{H} \hat{\mathbf{R}}_{x x}^{-1}(n) \mathbf{a}_{i}\right\}\left|\mathbf{c}^{H} \mathbf{a}_{i}^{*}\right|^{2}
$$

For binary phase-shift-keying (BPSK) signals, we assume that

$$
s_{i}(t)=\sigma_{i} e^{j\left(\pi \alpha_{i} t+\psi_{i}(t)\right)},
$$

where $\sigma_{i}$ and $\psi_{i}(t)$ denote the amplitude and the random phases equal to $\pm \pi / 2$ for the $i$ th SOI, respectively. Consequently, we have

$$
\widehat{r}_{s_{i} s_{i}^{*}}\left(\hat{\alpha}_{i}, 0\right)=-\sigma_{i}^{2} \operatorname{sinc}\left(\left(\hat{\alpha}_{i}-\alpha_{i}\right) T\right) .
$$

Consider the case of $\hat{\alpha}_{i}=\alpha_{i}+\Delta \alpha_{i}$. Then, (32) leads to

$$
\begin{aligned}
\frac{\partial \hat{r}_{s_{i} s_{i}^{*}}\left(\hat{\alpha}_{i}, 0\right)}{\partial \hat{\alpha}_{i}}= & -\sigma_{i}^{2} \frac{\partial \operatorname{sinc}\left(\Delta \alpha_{i} T\right)}{\partial \Delta \alpha_{i}} \\
= & -\sigma_{i}^{2} \frac{\pi \Delta \alpha_{i} T \cos \left(\pi \Delta \alpha_{i} T\right)-\sin \left(\pi \Delta \alpha_{i} T\right)}{\pi\left(\Delta \alpha_{i}\right)^{2} T} \\
\frac{\partial\left|\hat{r}_{s_{i} s_{i}^{*}}\left(\hat{\alpha}_{i}, 0\right)\right|^{2}}{\partial \hat{\alpha}_{i}}= & 2 \sigma_{i}^{4} \operatorname{sinc}\left(\Delta \alpha_{i} T\right) \\
& \times \frac{\pi \Delta \alpha_{i} T \cos \left(\pi \Delta \alpha_{i} n\right)-\sin \left(\pi \Delta \alpha_{i} T\right)}{\pi\left(\Delta \alpha_{i}\right)^{2} T} .
\end{aligned}
$$

Therefore, (30) becomes

$$
\begin{aligned}
\nabla_{\hat{\alpha}_{i}} C(\underline{\hat{\alpha}}, n) \approx & 2 \sigma_{i}^{4} \operatorname{sinc}\left(\Delta \alpha_{i} T\right)\left\{\mathbf{a}_{i}^{H} \hat{\mathbf{R}}_{x x}^{-1}(n) \mathbf{a}_{i}\right\}\left|\mathbf{c}^{H} \mathbf{a}_{i}^{*}\right|^{2} \\
& \times \frac{\pi \Delta \alpha_{i} T \cos \left(\pi \Delta \alpha_{i} T\right)-\sin \left(\pi \Delta \alpha_{i} T\right)}{\pi\left(\Delta \alpha_{i}\right)^{2} T} .
\end{aligned}
$$

Next, substituting (31) into (20) and performing some algebraic manipulations, we have

$$
\hat{\mathbf{R}}_{x u}^{\prime}\left(\hat{\alpha}_{i}, n\right) \approx \sigma_{i}^{2} \frac{\pi \Delta \alpha_{i} T \cos \left(\pi \Delta \alpha_{i} T\right)-\sin \left(\pi \Delta \alpha_{i} T\right)}{\pi\left(\Delta \alpha_{i}\right)^{2} T} \mathbf{a}_{i} \mathbf{a}_{i}^{T}
$$

+ SNOI-related terms.

At the time instant $n$, the time interval $T=n T_{s}$ and the estimated cycle frequencies are given by $\underline{\hat{\alpha}}(n)$. In order to make the influence due to SNOI negligible, it is appropriate to make sure that the relationship given by

$$
\left|\Delta \alpha_{i}(n)\left\{n T_{s}\right\}\right| \leq \frac{1}{2}, \quad i=1,2, \ldots, d,
$$

is kept, where $\Delta \alpha_{i}(n)=\hat{\alpha}_{i}(n)-\alpha_{i}$. Accordingly, the maximum singular value of $\hat{\mathbf{R}}_{x \dot{u}}\left(\hat{\alpha}_{i}(n), n\right)$ is approximately equal to

$$
M^{2} \sigma_{i}^{2}\left|\frac{\pi \Delta \alpha_{i}(n) T \cos \left(\pi \Delta \alpha_{i}(n) T\right)-\sin \left(\pi \Delta \alpha_{i}(n) T\right)}{\pi\left(\Delta \alpha_{i}(n)\right)^{2} T}\right|,
$$

where $M=\mathbf{a}_{i}^{H} \mathbf{a}_{i}$. As a result, the $i$ th step size parameter $\mu_{i}(n)$ of (22) is equal to

$$
\left\{M^{2} \sigma_{i}^{2}\left|\frac{\pi \Delta \alpha_{i}(n) T \cos \left(\pi \Delta \alpha_{i}(n) T\right)-\sin \left(\pi \Delta \alpha_{i}(n) T\right)}{\pi\left(\Delta \alpha_{i}(n)\right)^{2} T}\right|\right\}^{-P_{i}} .
$$

It follows from (21) that

$$
\Delta \underline{\alpha}(n+1)=\Delta \underline{\alpha}(n)+\Phi(n) \nabla_{\underline{\hat{\alpha}}(n)} C(\underline{\hat{\alpha}}(n), n)
$$


since $\underline{\hat{\alpha}}(n)=\underline{\alpha}+\Delta \underline{\alpha}(n)$. Substituting (34) and (38) into (39) yield

$$
\begin{aligned}
& \Delta \alpha_{i}(n+1)\left\{(n+1) T_{s}\right\} \\
&=\left\{\Delta \alpha_{i}(n)+\mu_{i}(n) \nabla_{\hat{\alpha}_{i}(n)} C\left(\hat{\alpha}_{i}(n), n\right)\right\}\left\{(n+1) T_{s}\right\} \approx \Delta \alpha_{i}(n) \\
&+\left\{2 M^{-2 P_{i}} \sigma_{i}^{4-2 P_{i}} \operatorname{sinc}\left(\Delta \alpha_{i}(n) n T_{s}\right)\left\{\mathbf{a}_{i}^{H} \hat{\mathbf{R}}_{x x}^{-1}(n) \mathbf{a}_{i}\right\}\left|\mathbf{c}^{H} \mathbf{a}_{i}^{*}\right|^{2}\right\} \\
& \cdot\left\{\frac{\pi \Delta \alpha_{i}(n) n T_{s} \cos \left(\pi \Delta \alpha_{i}(n) n T_{s}\right)-\sin \left(\pi \Delta \alpha_{i}(n) n T_{s}\right)}{\pi\left(\Delta \alpha_{i}(n)\right)^{2} n T_{s}}\right\}^{1-P_{i}} \\
& \times\left\{(n+1) T_{s}\right\} .
\end{aligned}
$$

Here, we prove that $\left|\Delta \alpha_{i}(n+1)\left\{(n+1) T_{s}\right\}\right| \leq 1 / 2$ if $\left|\Delta \alpha_{i}(n)\left\{n T_{s}\right\}\right| \leq 1 / 2$ to ensure the convergence of the proposed method. Substituting the extreme value $1 / 2$ for $\Delta \alpha_{i}(n)\left\{n T_{s}\right\}$ into (40) and performing some necessary algebraic manipulations yields

$$
\Delta \alpha_{i}(n+1)\left\{(n+1) T_{s}\right\} \approx \frac{n+1}{2 n}-\gamma_{i} \frac{n+1}{n^{P_{i}-1}},
$$

where $\gamma_{i}=2^{4-2 P_{i}} M^{-2 P_{i}} \sigma_{i}^{4-2 P_{i}} \pi^{P_{i}-2} T_{s}^{2-P_{i}}\left\{\mathbf{a}_{i}^{H} \hat{\mathbf{R}}_{x x}^{-1}(n) \mathbf{a}_{i}\right\}\left|\mathbf{c}^{H} \mathbf{a}_{i}^{*}\right|^{2}$. Hence, under the condition of $\left|\Delta \alpha_{i}(n)\left\{n T_{s}\right\}\right| \leq 1 / 2$, we have

$$
-1 \leq \frac{1}{2(n-1)}-\gamma_{i} \frac{n}{(n-1)^{P_{i}-1}} \leq 0 .
$$

It follows from (42) that

$$
\begin{aligned}
-1 & \leq \underbrace{\frac{1}{2 n}-\frac{(2 n-1)(n+1)}{2 n^{P_{i}}(n-1)^{2-P_{i}}}}_{B_{2}} \leq \frac{1}{2 n}-\gamma_{i} \frac{n+1}{n^{P_{i}-1}} \\
& \leq \underbrace{\frac{1}{2 n}-\frac{n+1}{2 n^{P_{i}}(n-1)^{2-P_{i}}}}_{B_{1}} \leq 0 .
\end{aligned}
$$

From (43), we note that $B_{1} \leq 0$ if $1 \leq P_{i} \leq 2, B_{2}>-1$ if $P_{i}=2$, and $B_{2}<-1$ if $P_{i}=1$. Accordingly, there exists some $P_{i}$ that can be appropriately chosen between 1 and 2 to make that $\left|\Delta \alpha_{i}(n+1)\left\{(n+1) T_{s}\right\}\right| \leq 1 / 2$. Similarly, substituting the other extreme value $-1 / 2$ for $\Delta \alpha_{i}(n)\left\{n T_{s}\right\}$ into (40), we have

$$
\Delta \alpha_{i}(n+1)\left\{(n+1) T_{s}\right\} \approx-\frac{n+1}{2 n}+\gamma_{i} \frac{n+1}{n^{P_{i}-1}}
$$

when $n$ is large enough. It is easy to show that

$$
\begin{aligned}
1 & \geq-\frac{1}{2 n}+\frac{(2 n-1)(n+1)}{2 n^{P_{i}}(n-1)^{2-P_{i}}} \geq-\frac{1}{2 n}+\gamma_{i} \frac{n+1}{n^{P_{i}-1}} \\
& \geq-\frac{1}{2 n}+\frac{i+1}{2 n^{P_{i}}(n-1)^{2-P_{i}}} \geq 0
\end{aligned}
$$

for $1 \leq P_{i} \leq 2$. Hence, there exists some $P_{i}$ between 1 and 2 such that $\left|\Delta \alpha_{i}(n+1)\left\{(n+1) T_{s}\right\}\right| \leq 1 / 2$ if $\left|\Delta \alpha_{i}(n)\left\{n T_{s}\right\}\right| \leq$ $1 / 2$. As a result, by exploiting the spectral conjugate selfcoherence property of the BPSK signals, the convergence of the proposed method can be guaranteed. However, for other types of cyclostationary signals such as QPSK signals or QAM signals, the convergence property may be different from that of BPSK signals. As a result, we have to find $\hat{r}_{s_{i} s_{i}^{*}}\left(\hat{\alpha}_{i}, 0\right)$ from the complex waveforms of QPSK signals or QAM signals. Then, we follow the similar procedure as described by (33) to (44) to show the convergence property for other types of cyclostationary signals such as QPSK signals or QAM signals.

\section{Simulation Examples}

Here, we present two simulation examples to show the effectiveness of the proposed method. For all simulations, we use a uniform linear array (ULA) with $M=10$ array elements and interelement spacing equal to $0.5 v$, where $v$ is the wavelength of the SOIs. Assume that the SOI and SNOI are BPSK signals with rectangular pulse shape. The SOI have the signal-to-noise ratio (SNR) and baud rate equal to $5 \mathrm{~dB}$ and 5/11, respectively. Two SNOIs with cycle frequencies equal to 4.6 and 7.8 impinge on the array from $-20^{\circ}$ and $40^{\circ}$ off broadside, respectively. Moreover, the SNOI have the interference-to-noise ratio (INR) and baud rate equal to $10 \mathrm{~dB}$ and $5 / 11$, respectively. The sampling interval $T_{s}$ is set to $0.1, L_{0}=100$, the control vector $\mathbf{c}$ is given by $\mathbf{c}=[10 \cdots 0]^{T}$, and $P_{i}=1.9, i=1,2, \ldots, d$ for simplicity.

Example 1. We present the output SINR versus the number of data snapshots for comparison. In this example, we consider the case of two SOIs $(d=2)$ with cycle frequencies $\underline{\alpha}=\{2,4\}$ impinging on the array from $10^{\circ}, 50^{\circ}$ off broadside. Moreover, we assume that the CFE of the SOIs is $\Delta \underline{\alpha}=\{0.01,-0.02\}$, that is, $\underline{\alpha}=\{2.01,3.98\}$. We observe from Figure 1 that there are periodic nulls for the original MLS-SCORE algorithm with CFE as the number data snapshots increases. In contrast, the proposed method can effectively cope with the performance degradation due to the CFE and provides the performance very close to that of the original MLS-SCORE algorithm without CFE.

Example 2. Consider that there are three SOIs $(d=3)$ two of the SOIs are the same as those used by Example 1, and the other SOI has different cycle frequency equal to 9 and is impinging on the array from $-20^{\circ}$ off broadside. Here, the CFE is set to $\Delta \underline{\alpha}=\{0.01,-0.01,0.02\}$, that is, $\underline{\hat{\alpha}}=$ $\{2.01,3.99,9.02\}$. As expected, we observe from Figure 2 that the performance degradation of the original MLS-SCORE algorithm becomes severer as the number data snapshots increases when the CFE exists. The proposed method works satisfactorily in the same circumstances.

\section{Conclusion}

This paper has evaluated the performance degradation of the original MLS-SCORE algorithm in the presence of CFE. An efficient method in conjunction with the MLS-SCORE algorithm has been proposed to overcome the CFE difficulty for achieving robust adaptive beamforming. Based on the proposed method, an appropriate estimate for each of the 


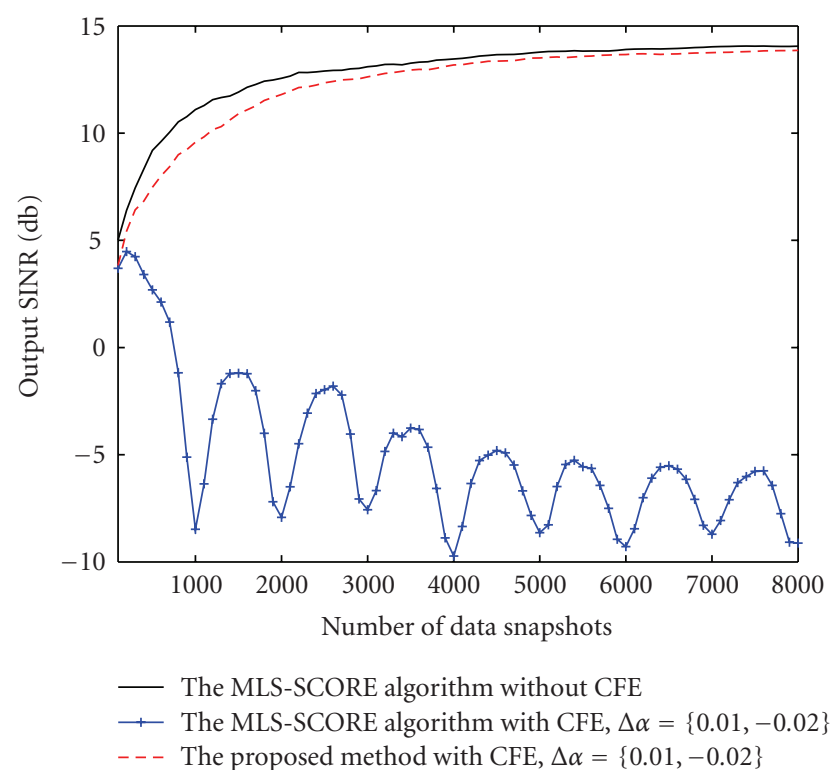

FIGURE 1: Output SINR versus number of data snapshots for Example 1.

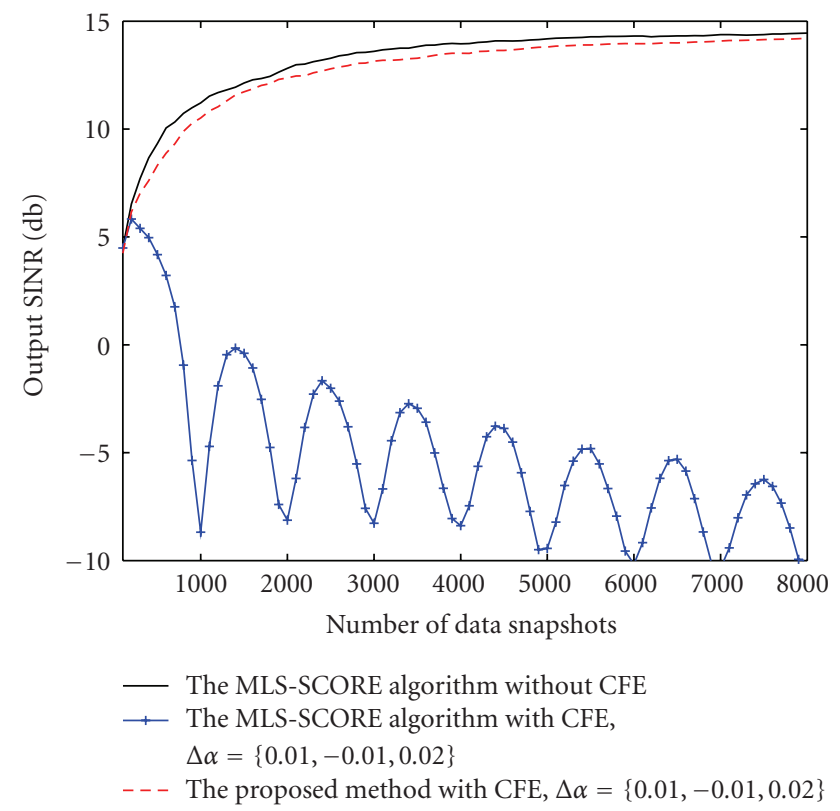

FIGURE 2: Output SINR versus number of data snapshots for Example 2.

cycle frequencies of the signals of interest is found iteratively by utilizing the steepest-descent method. The convergence of the proposed method has been shown for the case of using BPSK signals. Simulation results demonstrate that an adaptive beamforming using the proposed method can effectively cure the performance deterioration due to CFE and provides the performance very close to that of using the original MLS-SCORE algorithm without CFE.

\section{Acknowledgment}

This work was supported by the National Science Council of Taiwan under Grant NSC97-2221-E002-174-MY3.

\section{References}

[1] H. L. van Trees, Optimum Array Processing, Part IV of Detection, Estimation, and Modulation Theory, John Wiley \& Sons, New York, NY, USA, 2002.

[2] W. A. Gardner, A. Napolitano, and L. Paura, "Cyclostationarity: half a century of research," Signal Processing, vol. 86, no. 4, pp. 639-697, 2006.

[3] B. G. Agee, S. V. Schell, and W. A. Gardner, "Spectral selfcoherence restoral: a new approach to blind adaptive signal extraction using antenna arrays," Proceedings of the IEEE, vol. 78, no. 4, pp. 753-767, 1990.

[4] Q. Wu and K. M. Wong, "Blind adaptive beamforming for cyclostationary signals," IEEE Transactions on Signal Processing, vol. 44, no. 11, pp. 2757-2767, 1996.

[5] K. L. Du and M. N. S. Swamy, "A class of adaptive cyclostationary beamforming algorithms," Circuits, Systems, and Signal Processing, vol. 27, no. 1, pp. 35-63, 2008.

[6] J.- H. Lee and Y. T. Lee, "Robust adaptive array beamforming for cyclostationary signals under cycle frequency error," IEEE Transactions on Antennas and Propagation, vol. 47, no. 2, pp. 233-241, 1999.

[7] J.- H. Lee, Y. T. Lee, and W. H. Shih, "Efficient robust adaptive beamforming for cyclostationary signals," IEEE Transactions on Signal Processing, vol. 48, no. 7, pp. 1893-1901, 2000.

[8] J. Zhang, G. Liao, and J. Wang, "Robust direction finding for cyclostationary signals with cycle frequency error," Signal Processing, vol. 85, no. 12, pp. 2386-2393, 2005.

[9] J. T. Mayhan, "Area coverage adaptive nulling from geosynchronous satellites: phased arrays versus multiple-beam antennas," IEEE Transactions on Antennas and Propagation, vol. 34, no. 3, pp. 410-419, 1986.

[10] K. B. Yu, "Adaptive beamforming for satellite communication with selective earth coverage and jammer nulling capability," IEEE Transactions on Signal Processing, vol. 44, no. 12, pp. 3162-3166, 1996.

[11] J.-H. Lee and C.-C. Huang, "Blind adaptive beamforming for cyclostationary signals: a subspace projection approach," IEEE Antennas and Wireless Propagation Letters, vol. 8, pp. 14061409, 2009. 